\title{
PLANE STRAIN DEFORMATION IN A THERMOELASTIC MICROELONGATED SOLID WITH INTERNAL HEAT SOURCE
}

\author{
P. AILAWALIA* \\ Department of Applied Sciences and Humanities \\ M.M. University, Sadopur, Ambala City \\ Haryana, INDIA \\ E-mail: praveen_2117@rediffmail.com \\ S.K. SACHDEVA \\ Department of Applied Sciences \\ D.A.V Institute of Engineering and Technology \\ Jalandhar, Punjab, INDIA \\ (Research Scholar, Punjab Technical University, Kapurthala, Punjab, India) \\ E-mail: sunilsachdeva.daviet@gmail.com \\ D.S. PATHANIA \\ Department of Applied Sciences \\ Guru Nanak Dev Ji Engineering College \\ Ludhiana, Punjab, INDIA
}

\begin{abstract}
The purpose of this paper is to study the two dimensional deformation due to an internal heat source in a thermoelastic microelongated solid. A mechanical force is applied along an overlaying elastic layer of thickness $h$. The normal mode analysis has been applied to obtain the exact expressions for the displacement component, force stress, temperature distribution and microelongation. The effect of the internal heat source on the displacement component, force stress, temperature distribution and microelongation has been depicted graphically for Green-Lindsay (GL) theory of thermoelasticity.
\end{abstract}

Key words: thermoelasticity, microelongation, heat source, normal mode analysis.

\section{Introduction}

The dynamical interaction between the thermal and mechanical fields has great practical applications in modern aeronautics, astronatics, nuclear reactors, and high-energy particle accelerators. Classical elasticity is not adequate to model the behavior of materials possessing internal structure. Furthermore, the micropolar elastic model is more realistic than the purely elastic theory for studying the response of materials to external stimuli. Eringen and Suhubi (1964) and Suhubi and Eringen (1964) developed a nonlinear theory of microelastic solids. Later Eringen $(1965 ; 1966 ; 1996)$ developed a theory for the special class of micro-elastic materials and called it the "linear theory of micropolar elasticity". Under this theory, solids can undergo macro-deformations and micro-rotations. Eringen (1971) extended his work to include the axial stretch during the rotation of molecules and developed the theory of micro-polar elastic solid with stretch. The micropolar theory was extended to include thermal effects by Nowacki (1966), Eringen (1970), Tauchert et al. (1968), Tauchert (1971), Nowacki and Olszak (1974). One can refer to Dhaliwal and Singh (1987) for a

\footnotetext{
* To whom correspondence should be addressed
} 
review on the micropolar thermoelasticity and a historical survey of the subject, as well as to Eringen and Kafadar (1976) in "Continuum Physics" series in which the general theory of micromorphic media has been summed up.

There are two important generalized theories of thermoelasticity. The first is due to Lord and Shulman (1967). The second generalization of the coupled theory of elasticity is known as the theory of thermoelasticity with two relaxation times or the theory of temperature-rate-dependent thermoelasticity. Muller (1971), in the review of thermodynamics of thermoelastic solids, proposed an entropy production inequality, with the help of which he considered restrictions on a class of constitutive equations. A generalization of this inequality was proposed by Green and Laws (1972a). Green and Lindsay (1972b) obtained another version of the constitutive equations. These equations were also obtained independently and more explicitly by Suhubi (1975). This theory contains two constants that act as relaxation times and modify all the equations of coupled theory, not only the heat equations. The classical Fourier law of heat conduction is not violated if the medium under consideration has a centre of symmetry.

Barber (1984) studied thermoelastic displacements and stresses due to a heat source moving over the surface of a half plane. Sherief (1986) obtained components of stress and temperature distributions in a thermoelastic medium due to a continuous source. Dhaliwal et al. (1997) investigated thermoelastic interactions caused by a continuous line heat source in a homogeneous isotropic unbounded solid. Chandrasekharaiah and Srinath (1998) studied thermoelastic interactions due to a continuous point heat source in a homogeneous and isotropic unbounded body. Sharma et al. (2000) investigated the disturbance due to a time-harmonic normal point load in a homogeneous isotropic thermoelastic half-space. Sharma and Chauhan (2001) discussed mechanical and thermal sources in a generalized thermoelastic half-space. Sharma et al. (2004) investigated the steady-state response of an applied load moving with constant speed for infinite long time over the top surface of a homogeneous thermoelastic layer lying over an infinite half-space. Sarbani and Amitava (2004) studied the transient disturbance in half-space due to moving internal heat source under L-S model and obtained the solution for displacements in the transform domain. Aouadi (2006) studied thermomechanical interaction in a generalized thermo-microstretch elastic half space. Deswal and Choudhary (2008) studied a two-dimensional problem due to moving loads in generalized thermoelastic solid with diffusion. El Maghraby (2010) considered a two dimensional problem of a generalized thermoelastic half space under the action of body forces and subjected to thermal shock. Youssef (2010) solved the problem on a generalized thermoelastic infinite medium with a spherical cavity subjected to a moving heat source. Shaw and Mukhopadhyay $(2012 ; 2013)$ discussed a couple of problems in a thermoelactic microelongated medium subjected to a heat source.

In the present problem the authors have discussed deformation due to an internal heat source in a thermoelastic microelongated solid with an overlaying elastic layer of thickness $h$. A mechanical force of constant magnitude is applied along the layer. The normal mode analysis is used to obtain the exact expressions for the considered variables. The distributions of the considered variables are then represented graphically for Green Lindsay (GL) theory of thermoelasticity.

The constitutive equation for a homogeneous, isotropic, microelongated, thermoelastic solid are

$$
\begin{aligned}
& \sigma_{k l}=-\beta_{0}\left(1+t_{l} \delta_{2 k} \frac{\partial}{\partial t}\right) T \delta_{k l}+\lambda_{0} \delta_{k l} \phi+\lambda \delta_{k l} u_{r, r}+\mu\left(u_{k, l}+u_{l, k}\right), \\
& m_{k}=a_{0} \phi_{, k} \\
& s-t=-\beta_{l}\left(1+t_{l} \delta_{2 k} \frac{\partial}{\partial t}\right) T+\lambda_{l} \phi+\lambda_{0} u_{k, k} \\
& q_{k}=\frac{K}{T_{0}} T_{, k} .
\end{aligned}
$$


The field equation of motion according to Eringen (1999), Kiris and Inan (2007) and the heat conduction equation according to De Cicco and Nappa (1999) for the displacement, microelongation and temperature changes are

$$
\begin{aligned}
& -\beta_{0}\left(1+t_{1} \delta_{2 k} \frac{\partial}{\partial t}\right) T_{, i}+\lambda_{0} \phi_{, i}+(\lambda+\mu) u_{j, i j}+\mu u_{i, j j}=\rho \ddot{u}_{i}, \\
& a_{0} \phi_{, i i}+\beta_{1}\left(1+t_{l} \delta_{2 k} \frac{\partial}{\partial t}\right) T+\lambda_{l} \phi-\lambda_{0} u_{j, j}=\frac{1}{2} \rho j_{0} \ddot{\phi}, \\
& K \nabla^{2} T-\rho C_{E}\left(1+t_{0} \delta_{1 k} \frac{\partial}{\partial t}\right) \dot{T}=\beta_{0} T_{0}\left(1+t_{0} \delta_{l k} \frac{\partial}{\partial t}\right) \dot{u}_{k, k}+\beta_{1} T_{0} \dot{\phi}-\rho\left(1+t_{0} \delta_{1 k} \frac{\partial}{\partial t}\right) Q .
\end{aligned}
$$

We have considered a homogenous, microelongated, isotropic, infinite, thermoelastic body at a uniform reference temperature $T_{0}$ in the presence of an internal heat source $Q$ in the $x y$-plane with displacement vector $\boldsymbol{u}=(u, v, 0)$, i.e., two dimensional disturbance of medium is assumed.

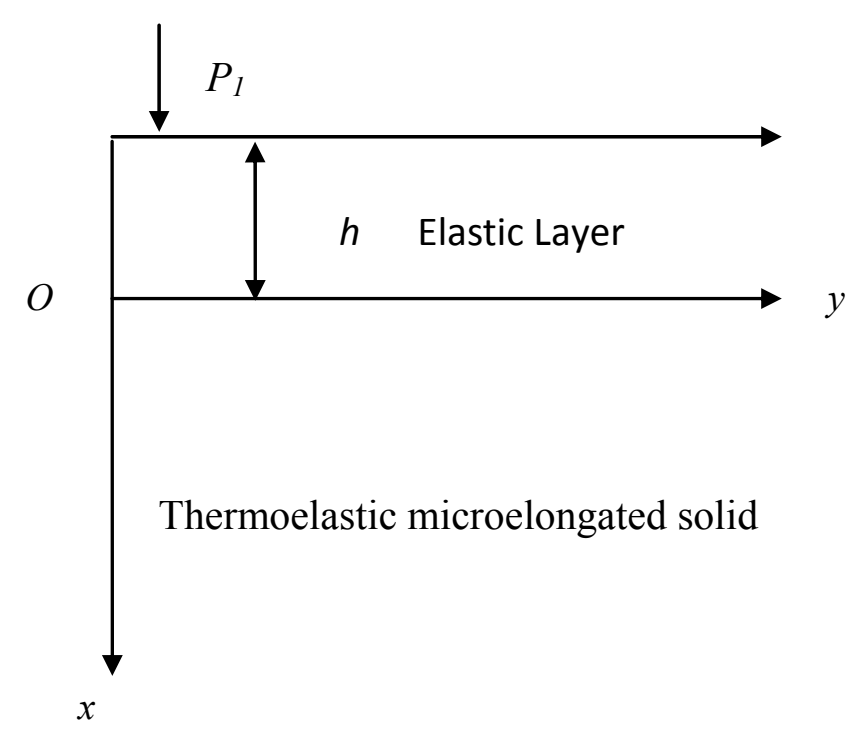

Fig.1. Geometry of the problem.

Hence, Eqs (1.5)-(1.7) become

$$
\begin{aligned}
& -\beta_{0}\left(1+t_{l} \delta_{2 k} \frac{\partial}{\partial t}\right) \frac{\partial T}{\partial x}+\lambda_{0} \frac{\partial \phi}{\partial x}+(\lambda+2 \mu) \frac{\partial^{2} u}{\partial x^{2}}+(\lambda+\mu) \frac{\partial^{2} v}{\partial x \partial y}+\mu \frac{\partial^{2} u}{\partial y^{2}}=\rho \frac{\partial^{2} u}{\partial t^{2}} \\
& -\beta_{0}\left(1+t_{l} \delta_{2 k} \frac{\partial}{\partial t}\right) \frac{\partial T}{\partial y}+\lambda_{0} \frac{\partial \phi}{\partial y}+\mu \frac{\partial^{2} v}{\partial x^{2}}+(\lambda+\mu) \frac{\partial^{2} u}{\partial x \partial y}+(\lambda+2 \mu) \frac{\partial^{2} v}{\partial y^{2}}=\rho \frac{\partial^{2} v}{\partial t^{2}}
\end{aligned}
$$




$$
\begin{aligned}
& a_{0} \nabla^{2} \phi+\beta_{1}\left(1+t_{l} \delta_{2 k} \frac{\partial}{\partial t}\right) T+\lambda_{l} \phi-\lambda_{0}\left(\frac{\partial u}{\partial x}+\frac{\partial v}{\partial y}\right)=\frac{1}{2} \rho j_{0} \frac{\partial^{2} \phi}{\partial t^{2}}, \\
& K \nabla^{2} T-\rho C_{E}\left(1+t_{0} \delta_{1 k} \frac{\partial}{\partial t}\right) \frac{\partial T}{\partial t}= \\
& =\beta_{0} T_{0}\left(\frac{\partial}{\partial t}+t_{0} \delta_{l k} \frac{\partial^{2}}{\partial t^{2}}\right)\left(\frac{\partial u}{\partial x}+\frac{\partial v}{\partial y}\right)+\beta_{1} T_{0} \frac{\partial \phi}{\partial t}-\rho\left(1+t_{0} \delta_{l k} \frac{\partial}{\partial t}\right) Q .
\end{aligned}
$$

Constitutive stress components are

$$
\begin{aligned}
& \sigma_{x x}=-\beta_{0} T+\lambda_{0} \phi+(\lambda+2 \mu) \frac{\partial u}{\partial x}+\lambda \frac{\partial v}{\partial y}, \\
& \sigma_{y y}=-\beta_{0}\left(1+t_{1} \frac{\partial}{\partial t}\right) T+\lambda_{0} \phi+\lambda \frac{\partial u}{\partial x}+(\lambda+2 \mu) \frac{\partial v}{\partial y}, \\
& \sigma_{x y}=\mu\left(\frac{\partial u}{\partial x}+\frac{\partial v}{\partial y}\right) .
\end{aligned}
$$

The equations of motion and stress components in an elastic medium are Ewing et al. (1957)

$$
\begin{aligned}
& \left(\lambda^{e}+\mu^{e}\right) \nabla\left(\nabla \cdot \boldsymbol{u}^{e}\right)+\mu^{e} \nabla^{2} \boldsymbol{u}^{e}=\rho^{e} \frac{\partial^{2} \boldsymbol{u}^{e}}{\partial t^{2}}, \\
& \sigma_{i j}^{e}=\lambda^{e}\left(\nabla \cdot u^{e}\right) \delta_{i j}+\mu^{e}\left(u_{i, j}^{e}+u_{j, i}^{e}\right) .
\end{aligned}
$$

For convenience the following non-dimensional variables are used

$$
\begin{aligned}
& x^{\prime}=\frac{\omega^{*}}{c_{1}} x, \quad y^{\prime}=\frac{\omega^{*}}{c_{1}} y, \quad u^{\prime}=\frac{\omega^{*} \rho c_{1}}{\beta_{0} T_{0}} u, \quad v^{\prime}=\frac{\omega^{*} \rho c_{1}}{\beta_{0} T_{0}} v, \quad t^{\prime}=\omega^{*} t, \quad t_{0}^{\prime}=\omega^{*} t_{0}, \quad t_{1}^{\prime}=\omega^{*} t_{1}, \\
& \sigma_{i j}^{\prime}=\frac{\sigma_{i j}}{\beta_{0} T_{0}}, \quad \phi^{\prime}=\frac{\lambda_{0}}{\beta_{0} T_{0}} \phi, \quad \sigma_{i j}^{e^{\prime}}=\frac{\sigma_{i j}^{e}}{\beta_{0} T_{0}}, \quad P_{1}^{\prime}=\frac{P_{1}}{\beta_{0} T_{0}}, \quad T^{\prime}=\frac{T}{T_{0}}, \quad Q^{\prime}=\frac{1}{\omega^{*} c_{1}^{2}} Q,
\end{aligned}
$$

where,

$$
\omega^{*}=\frac{\rho c_{l}^{2} C_{E}}{K}, \quad c_{1}^{2}=\frac{\lambda+2 \mu}{\rho} .
$$

Using the above non dimensional variables, Eqs (1.8)-(1.14) reduce to (after dropping superscripts)

$$
\frac{\partial^{2} u}{\partial t^{2}}=-\left(1+t_{1} \delta_{2 k} \frac{\partial}{\partial t}\right) \frac{\partial T}{\partial x}+\frac{\partial \phi}{\partial x}+h_{1} \frac{\partial^{2} u}{\partial x^{2}}+h_{2} \frac{\partial^{2} v}{\partial x \partial y}+h_{3} \frac{\partial^{2} u}{\partial y^{2}}
$$




$$
\begin{aligned}
& \frac{\partial^{2} v}{\partial t^{2}}=-\left(1+t_{1} \delta_{2 k} \frac{\partial}{\partial t}\right) \frac{\partial T}{\partial y}+\frac{\partial \phi}{\partial y}+h_{3} \frac{\partial^{2} v}{\partial x^{2}}+h_{2} \frac{\partial^{2} u}{\partial x \partial y}+h_{1} \frac{\partial^{2} v}{\partial y^{2}} \\
& \nabla^{2} \phi+h_{4}\left(1+t_{1} \delta_{2 k} \frac{\partial}{\partial t}\right) T-h_{5} \phi-h_{6}\left(\frac{\partial u}{\partial x}+\frac{\partial v}{\partial y}\right)=h_{7} \frac{\partial^{2} \phi}{\partial t^{2}} \\
& \nabla^{2} T-h_{8}\left(1+t_{0} \delta_{1 k} \frac{\partial}{\partial t}\right) \frac{\partial T}{\partial t}= \\
& =h_{9}\left(\frac{\partial}{\partial t}+t_{0} \delta_{1 k} \frac{\partial^{2}}{\partial t^{2}}\right)\left(\frac{\partial u}{\partial x}+\frac{\partial v}{\partial y}\right)+h_{10} \frac{\partial \phi}{\partial t}-h_{11}\left(1+t_{0} \delta_{1 k} \frac{\partial}{\partial t}\right) \\
& \sigma_{x x}=-T+\phi+h_{1} \frac{\partial u}{\partial x}+h_{12} \frac{\partial v}{\partial y}, \\
& \sigma_{y y}=-\left(1+t_{1} \frac{\partial}{\partial t}\right) T+\phi+h_{12} \frac{\partial u}{\partial x}+h_{1} \frac{\partial v}{\partial y} \\
& \sigma_{x y}=h_{3}\left(\frac{\partial u}{\partial x}+\frac{\partial v}{\partial y}\right), \\
& \left.x^{2}\right)
\end{aligned}
$$

where, $\quad\left(h_{1}, h_{2}, h_{3}\right)=\frac{(\lambda+2 \mu, \lambda+\mu, \mu)}{\rho c_{1}^{2}}, \quad h_{4}=\frac{\beta_{1} \lambda_{0} c_{1}^{2}}{a_{0} \omega^{*} \beta_{0}}, \quad h_{5}=\frac{\lambda_{1} c_{1}^{2}}{a_{0} \omega^{*}}, \quad h_{6}=\frac{\lambda_{0}{ }^{2}}{\rho a_{0} \omega^{*}}$,

$$
\begin{aligned}
& h_{7}=\frac{\rho j_{0} \omega^{*} c_{1}^{2}}{2 a_{0}}, \quad h_{8}=\frac{\rho C_{E} c_{1}^{2}}{K \omega^{*}}, \quad h_{9}=\frac{\beta_{0}^{2} T_{0}}{K \omega^{*} \rho}, \quad h_{10}=\frac{\beta_{0} \beta_{1} T_{0} c_{1}^{2}}{K \omega^{*} \lambda_{0}}, \quad h_{11}=\frac{\rho c_{1}^{4}}{K \omega^{*} T_{0}}, \\
& h_{12}=\frac{\lambda}{\rho c_{1}^{2}} .
\end{aligned}
$$

\section{Normal mode analysis}

The solution of the considered physical variables can be decomposed in terms of normal mode and can be considered in the following form

$$
\left(u, v, T, \phi, \sigma_{i j}, u^{e}, v^{e}, \sigma_{i j}^{e}, Q\right)(x, y, t)=\left(u^{*}, v^{*}, T^{*}, \phi^{*}, \sigma_{i j}^{*}, u^{e^{*}}, v^{e^{*}}, \sigma_{i j}^{e^{*}}, Q^{*}\right)(x) e^{\omega t+i b y}
$$

where $\omega$ is the complex frequency, $b$ is the wave number in the $y$-direction and $u^{*}(x), v^{*}(x), T^{*}(x), \phi^{*}(x)$, $\sigma_{i j}^{*}(x), u^{e^{*}}(x), v^{e^{*}}(x), \sigma_{i j}^{e^{*}}(x), Q^{*}$ are the amplitudes of field quantities.

Using normal mode in Eqs (1.17)-(1.23), we get 


$$
\begin{aligned}
& \left(h_{1} D^{2}-A_{1}\right) u^{*}+i b h_{2} D v^{*}-A_{2} D T^{*}+D \phi^{*}=0 \\
& i b h_{2} D u^{*}+\left(h_{3} D^{2}-A_{3}\right) v^{*}-i b A_{2} T^{*}+i b \phi^{*}=0 \\
& -h_{6} D u^{*}-i b h_{6} v^{*}+A_{2} h_{4} T^{*}+\left(D^{2}-A_{4}\right) \phi^{*}=0 \\
& -h_{9} A_{6} D u^{*}-i b h_{9} A_{6} v^{*}+\left(D^{2}-A_{7}\right) T^{*}-h_{10} \omega \phi^{*}=-h_{11} A_{5} Q^{*}, \\
& \sigma_{x x}^{*}=-T^{*}+\phi^{*}+h_{1} D u^{*}+i b h_{12} v^{*}, \\
& \sigma_{x y}^{*}=h_{3}\left(i b u^{*}+D v^{*}\right) \\
& \sigma_{y y}^{*}=-A_{1} T^{*}+\phi^{*}+h_{12} D u^{*}+i b h_{1} v^{*}
\end{aligned}
$$

where

$$
\begin{aligned}
& A_{1}=\omega^{2}+h_{3} b^{2}, \quad A_{2}=\left(1+t_{1} \delta_{2 k} \omega\right), \quad A_{3}=\omega^{2}+h_{1} b^{2}, \quad A_{4}=b^{2}+h_{5}+h_{7} \omega^{2}, \\
& A_{5}=\left(1+t_{0} \delta_{1 k} \omega\right), \quad A_{6}=\omega\left(1+t_{0} \delta_{1 k} \omega\right), \quad A_{7}=b^{2}+h_{8} A_{5} \omega, \quad D=\frac{d}{d x} .
\end{aligned}
$$

Eliminating $v^{*}(x), T^{*}(x), \phi^{*}(x)$ from Eqs (2.1)-(2.4), we get the following eight order differential equation for $u^{*}(x)$ as

$$
\left(D^{8}+A D^{6}+B D^{4}+C D^{2}+E\right) u^{*}(x)=R Q^{*}
$$

where,

$$
\begin{aligned}
& A=\frac{-1}{h_{1} h_{3}}\left[h_{1} h_{3}\left(A_{4}+A_{7}\right)-h_{1} A_{3}+h_{3} A_{1}+h_{3} h_{6}+A_{2} h_{3} h_{9} A_{6}+b^{2} h_{2}^{2}\right], \\
& B=\frac{-1}{h_{1} h_{3}}\left[-h_{1} A_{2} h_{4} h_{10} h_{3} \omega+h_{1} h_{3} A_{4} A_{7}+h_{1} A_{3}\left(A_{4}+A_{7}\right)-h_{1} b^{2} A_{2} A_{6} h_{9}+\right. \\
& +b^{2} h_{1} A_{6}-A_{1} h_{3}\left(A_{4}+A_{7}\right)+A_{1} A_{3}-b^{2} h_{2}^{2}\left(A_{4}+A_{7}\right)+h_{3} h_{6} h_{10} A_{2} \omega+ \\
& \left.-h_{3} h_{9} A_{2} A_{4} A_{6}-h_{9} A_{2} A_{3} A_{6}-h_{3} h_{6} A_{7}-h_{3} h_{4} h_{9} A_{2} A_{6}-A_{3} h_{6}\right], \\
& C=\frac{-1}{h_{1} h_{3}}\left[A_{2} A_{3} h_{1} h_{4} h_{10} \omega+A_{3} A_{4} A_{7} h_{1}-b^{2} h_{1} h_{6} h_{10} A_{2} \omega+b^{2} h_{1} h_{9} A_{2} A_{4} A_{6}+\right. \\
& -b^{2} h_{1} h_{6} A_{7}-b^{2} h_{1} h_{4} h_{9} A_{2} A_{6}+A_{1} A_{2} h_{3} h_{4} h_{10} \omega^{2}-h_{3} A_{1} A_{4} A_{7}+A_{1} A_{3}\left(A_{4}+A_{7}\right)+ \\
& +b^{2} A_{1} A_{2} A_{6} h_{9}-b^{2} h_{6} A_{1}+b^{2} h_{2}^{2} A_{2} h_{4} h_{10} \omega+b^{2} h_{2}^{2} A_{4} A_{7}+ \\
& \left.-2 b^{2} A_{7} h_{2} h_{6}-2 b^{2} A_{2} A_{6} h_{2} h_{4} h_{9}-h_{6} h_{10} A_{2} A_{3} \omega+A_{2} A_{3} A_{4} A_{6} h_{9}+A_{3} A_{7} h_{6}+A_{2} A_{3} A_{6} h_{4} h_{9}\right],
\end{aligned}
$$




$$
\begin{aligned}
& E=\frac{-1}{h_{1} h_{3}}\left(-h_{4} h_{10} A_{1} A_{2} A_{3} \omega-A_{1} A_{3} A_{4} A_{7}+b^{2} h_{6} h_{10} A_{1} A_{2} \omega+\right. \\
& \left.-b^{2} h_{9} A_{1} A_{2} A_{4} A_{6}+b^{2} h_{6} A_{1} A_{7}+b^{2} h_{4} h_{9} A_{1} A_{2} A_{6}\right) \\
& R=b^{2} h_{11} A_{1} A_{2} A_{4} A_{5}\left(h_{4}-A_{4}\right) .
\end{aligned}
$$

In a similar manner we can show that $v^{*}(x), \theta^{*}(x), \phi^{*}(x)$ satisfies the equation

$$
\left(D^{8}+A D^{6}+B D^{4}+C D^{2}+E\right)\left(v^{*}(x), \theta^{*}(x), \phi^{*}(x)\right)=R Q^{*},
$$

which can be factorized as follows

$$
\left(D^{2}-k_{1}^{2}\right)\left(D^{2}-k_{2}^{2}\right)\left(D^{2}-k_{3}^{2}\right)\left(D^{2}-k_{4}^{2}\right) u^{*}(x)=R Q^{*}
$$

The series solution of Eq.(2.8) has the form

$$
\begin{aligned}
& u^{*}(x)=\sum_{n=1}^{4}\left[M_{n}(b, \omega) e^{-k_{n} x}\right]+S, \\
& v^{*}(x)=\sum_{n=1}^{4}\left[M_{n}^{\prime}(b, \omega) e^{-k_{n} x}\right]-S_{1}, \\
& T^{*}(x)=\sum_{n=1}^{4}\left[M_{n}^{\prime \prime}(b, \omega) e^{-k_{n} x}\right]-S_{2}, \\
& \phi^{*}(x)=\sum_{n=1}^{4}\left[M_{n}^{\prime \prime \prime}(b, \omega) e^{-k_{n} x}\right]-S_{3}
\end{aligned}
$$

where $M_{n}(b, \omega), M_{n}^{\prime}(b, \omega), M_{n}^{\prime \prime}(b, \omega), M_{n}^{\prime \prime \prime}(b, \omega)$ are specific functions depending upon $b$, $\omega$ and $k_{n}^{2}$, $n=1,2,3,4$ are the roots of characteristic Eq.(2.10).

where, $S=\frac{R Q^{*}}{E}$.

Using Eqs (2.11)-(2.14) in Eqs (2.1)-(2.4), we get

$$
\begin{aligned}
& M_{n}^{\prime}(b, \omega)=H_{1 n} M_{n}(b, \omega), \\
& M_{n}^{\prime}(b, \omega)=H_{2 n} M_{n}(b, \omega), \\
& M_{n}^{\prime \prime \prime}(b, \omega)=H_{3 n} M_{n}(b, \omega),
\end{aligned}
$$




$$
S_{I}=i b A_{l} S, \quad S_{2}=i b A_{1}\left(A_{3} A_{4}-b^{2} h_{6}\right) S, \quad S_{3}=A_{1} S .
$$

Thus we have

$$
\begin{aligned}
& v^{*}(x)=\sum_{n=1}^{4}\left[H_{1 n} M_{n}(b, \omega) e^{-k_{n} x}\right]-S_{1}, \\
& T^{*}(x)=\sum_{n=1}^{4}\left[H_{2 n} M_{n}(b, \omega) e^{-k_{n} x}\right]-S_{2}, \\
& \phi^{*}(x)=\sum_{n=1}^{4}\left[H_{3 n} M_{n}(b, \omega) e^{-k_{n} x}\right]-S_{3}, \\
& \sigma_{x x}^{*}(x)=\sum_{n=1}^{4}\left[H_{4 n} M_{n}(b, \omega) e^{-k_{n} x}\right]+S_{4}, \\
& \sigma_{x y}^{*}(x)=\sum_{n=1}^{4}\left[H_{5 n} M_{n}(b, \omega) e^{-k_{n} x}\right]+S_{5}, \\
& \sigma_{y y}^{*}(x)=\sum_{n=1}^{4}\left[H_{6 n} M_{n}(b, \omega) e^{-k_{n} x}\right]+S_{6}
\end{aligned}
$$

where

$$
\begin{aligned}
& H_{1 n}=\frac{i b\left[\left(h_{1}-h_{2}\right) k_{n}^{2}-A_{1}\right]}{\left[\left(A_{3}-b^{2} h_{2}\right) k_{n}-h_{3} k_{n}^{3}\right]}, \\
& H_{2 n}=\frac{\left[h_{3} k_{n}^{4}-\left(A_{4} h_{3}+A_{3}\right) k_{n}^{2}+\left(A_{3} A_{4}-b^{2} h_{6}\right)\right] H_{1 n}-i b\left[h_{2} k_{n}^{3}-\left(h_{2} A_{4}-h_{6}\right) k_{n}\right]}{i b\left[A_{2}\left(k_{n}^{2}-A_{4}\right)+A_{2} h_{4}\right]}, \\
& H_{3 n}=\frac{\left(h_{1} k_{n}^{2}-A_{1}-i b h_{2} k_{n} H_{1 n}+A_{2} k_{n} H_{2 n}\right)}{k_{n}} \\
& H_{4 n}=i b h_{12} H_{1 n}-H_{2 n}+H_{3 n}-h_{1} k_{n}, \\
& H_{5 n}=h_{3}\left(i b-k_{n} H_{1 n}\right), \\
& H_{6 n}=i b h_{1} H_{1 n}-A_{1} H_{2 n}+H_{3 n}-h_{12} k_{n},
\end{aligned}
$$




$$
S_{4}=-\left(S_{2}+S_{3}+i b h_{12} S_{1}\right), \quad S_{5}=i b h_{3} S, \quad S_{6}=-\left(A_{1} S_{2}+S_{3}+i b h_{1} S_{1}\right)
$$

Similarly for medium II (i.e., elastic layer), the solutions are of the form

$$
\begin{aligned}
& u^{e^{*}}(x)=\sum_{n=1}^{2}\left[R_{n}(b, \omega) e^{-r_{n} x}\right]+\sum_{n=1}^{2}\left[R_{n+2}(b, \omega) e^{r_{n} x}\right], \\
& v^{e^{*}}(x)=\sum_{n=1}^{2}\left[R_{n}^{\prime}(b, \omega) e^{-r_{n} x}\right]+\sum_{n=1}^{2}\left[R_{n+2}^{\prime}(b, \omega) e^{r_{n} x}\right]
\end{aligned}
$$

where, $R_{n}(b, \omega), R_{n+2}(b, \omega)$ and $R_{n}^{\prime}(b, \omega), R_{n+2}^{\prime}(b, \omega)$ are specific functions depending upon $b, \omega$ and $r_{n}^{2}$, $n=1,2$ are the roots of characteristic equation

$$
\left(D^{4}-G D^{2}+L\right) u^{e^{*}}(x)=0
$$

where, $\quad G=\frac{b^{2} l_{1}^{2}+l_{1} \omega^{2}+b^{2} l_{3}^{2}+l_{3} \omega^{2}-b^{2} l_{2}^{2}}{l_{1} l_{3}}$,

$$
L=\frac{b^{4} l_{1} l_{3}+b^{2} l_{3} \omega^{2}+b^{2} l_{1} \omega^{2}+\omega^{4}}{l_{1} l_{3}},
$$

and

$$
l_{1}=\frac{\lambda^{e}+2 \mu^{e}}{\rho^{e} c_{1}^{2}}, \quad l_{2}=\frac{\lambda^{e}+\mu^{e}}{\rho^{e} c_{1}^{2}}, \quad l_{3}=\frac{\mu^{e}}{\rho^{e} c_{1}^{2}} .
$$

Thus we have

$$
\begin{aligned}
& v^{e^{*}}(x)=\sum_{n=1}^{2}\left[L_{1 n} R_{n}(b, \omega) e^{-r_{n} x}\right]+\sum_{n=1}^{2}\left[L_{l(n+2)} R_{n+2}(b, \omega) e^{r_{n} x}\right], \\
& \sigma_{x x}^{e^{*}}(x)=\sum_{n=1}^{2}\left[L_{2 n} R_{n}(b, \omega) e^{-r_{n} x}\right]+\sum_{n=1}^{2}\left[L_{2(n+2)} R_{n+2}(b, \omega) e^{r_{n} x}\right], \\
& \sigma_{y y}^{e^{*}}(x)=\sum_{n=1}^{2}\left[L_{3 n} R_{n}(b, \omega) e^{-r_{n} x}\right]+\sum_{n=1}^{2}\left[L_{3(n+2)} R_{n+2}(b, \omega) e^{r_{n} x}\right], \\
& \sigma_{x y}^{e^{*}}(x)=\sum_{n=1}^{2}\left[L_{4 n} R_{n}(b, \omega) e^{-r_{n} x}\right]+\sum_{n=1}^{2}\left[L_{4(n+2)} R_{n+2}(b, \omega) e^{r_{n} x}\right]
\end{aligned}
$$

where 


$$
\begin{array}{ll}
L_{1 n}=\frac{l_{1} r_{n}^{2}-b^{2} l_{3}-\omega^{2}}{i b l_{2} r_{n}}, & L_{l(n+2)}=\frac{l_{1} r_{n}^{2}-b^{2} l_{3}-\omega^{2}}{-i b l_{2} r_{n}}, \\
L_{2 n}=\frac{\left(\lambda^{e}+2 \mu^{e}\right)\left(-r_{n}\right)+i b L_{1 n}}{\mu}, & L_{2(n+2)}=\frac{\left(\lambda^{e}+2 \mu^{e}\right)\left(r_{n}\right)+i b L_{l(n+2)}}{\mu}, \\
L_{3 n}=\frac{\lambda^{e}\left(-r_{n}\right)+i b\left(\lambda^{e}+2 \mu^{e}\right) L_{1 n}}{\mu}, & L_{3(n+2)}=\frac{\lambda^{e}\left(r_{n}\right)+i b\left(\lambda^{e}+2 \mu^{e}\right) L_{1(n+2)}}{\mu}, \\
L_{4 n}=\frac{i b \mu^{e}-r_{n} \mu^{e} L_{l n}}{\mu}, & L_{4(n+2)}=\frac{i b \mu^{e}+r_{n} \mu^{e} L_{l(n+2)}}{\mu} .
\end{array}
$$

\section{Applications}

In this section we determine the parameter $M_{n}$ and $R_{n} ;(n=1,2,3,4)$. In the physical problem the constants $M_{n}$ and $R_{n} ;(n=1,2,3,4)$ have to be selected such that boundary conditions at the surface are

$$
\begin{aligned}
& \sigma_{x x}=\sigma_{x x}^{e}-P_{1} e^{\omega t+i b y} \quad \text { at } \quad x=-h ; \quad \sigma_{x y}=0 \quad \text { at } \quad x=-h ; \\
& \sigma_{x x}=\sigma_{x x}^{e} \quad \text { at } \quad x=0 ; \quad \sigma_{x y}=\sigma_{x y}^{e} \quad \text { at } \quad x=0 ; \quad u=u^{e} \quad \text { at } \quad x=0 ; \\
& v=v^{e} \quad \text { at } \quad x=0 ; \quad \phi=0 \quad \text { at } \quad x=0 ; \quad \frac{\partial T}{\partial x}=0 \quad \text { at } \quad x=0
\end{aligned}
$$

where $P_{l}$ is the magnitude of mechanical force.

Using the expressions of $\sigma_{x x}, \sigma_{x x}^{e}, \sigma_{x y}, \sigma_{x y}^{e}, u, u^{e}, v, v^{e}, \phi$ and $T$ from Eqs (2.18)-(2.22) and (2.24), (2.27)-(2.30) in the above boundary conditions (3.1), gives the following equations satisfied by the parameters

$$
\begin{aligned}
& \sum_{n=1}^{4}\left[H_{4 n} M_{n}\right] e^{k_{n} h}-\sum_{n=1}^{2}\left[L_{2 n} R_{n}\right] e^{r_{n} h}-\sum_{n=1}^{2}\left[L_{2(n+2)} R_{n+2}\right] e^{-r_{n} h}=-P_{1}-S_{4}, \\
& \sum_{n=1}^{4}\left[H_{5 n} M_{n}\right] e^{k_{n} h}=-S_{5}, \\
& \sum_{n=1}^{4}\left[H_{4 n} M_{n}\right]-\sum_{n=1}^{2}\left[L_{2 n} R_{n}\right]-\sum_{n=1}^{2}\left[L_{2(n+2)} R_{n+2}\right]=-S_{4}, \\
& \sum_{n=1}^{4}\left[H_{5 n} M_{n}\right]-\sum_{n=1}^{2}\left[L_{4 n} R_{n}\right]-\sum_{n=1}^{2}\left[L_{4(n+2)} R_{n+2}\right]=-S_{5}, \\
& \sum_{n=1}^{4}\left[M_{n}\right]-\sum_{n=1}^{2}\left[R_{n}\right]-\sum_{n=1}^{2}\left[R_{n+2}\right]=-S,
\end{aligned}
$$




$$
\begin{aligned}
& \sum_{n=1}^{4}\left[H_{l n} M_{n}\right]-\sum_{n=1}^{2}\left[L_{l n} R_{n}\right]-\sum_{n=1}^{2}\left[L_{l(n+2)} R_{n+2}\right]=S_{l}, \\
& \sum_{n=1}^{4}\left[H_{3 n} M_{n}\right]=S_{3}, \\
& \sum_{n=1}^{4}\left[H_{2 n} k_{n} M_{n}\right]=0 .
\end{aligned}
$$

After solving the above non homogenous system of eight equations, we get the values of constants $M_{n}$ and $R_{n} ;(n=1,2,3,4)$ and hence we obtain the component of normal displacement, normal force stress, temperature distribution and microelongation due to the internal heat source in a thermoelastic microelongated solid with an overlaying elastic layer.

\subsection{Special case}

Letting $\phi \rightarrow 0$, we obtain the results for a generalized thermoelastic solid (TS).

\section{Numerical results and discussions}

For numerical computations, we consider the values of physical constants for a thermoelastic microelongated solid as given by Shaw and Mukhopadhyay (2013)

$$
\begin{aligned}
& \lambda=7.59 \times 10^{10} \mathrm{~N} / \mathrm{m}^{2}, \quad \mu=1.89 \times 10^{10} \mathrm{~N} / \mathrm{m}^{2}, \quad a_{0}=0.61 \times 10^{-10} \mathrm{~N}, \\
& \rho=2.19 \times 10^{3} \mathrm{~kg} / \mathrm{m}^{3}, \quad \beta_{1}=0.05 \times 10^{5} \mathrm{~N} / \mathrm{m}^{2} \mathrm{~K}, \quad \beta_{0}=0.05 \times 10^{5} \mathrm{~N} / \mathrm{m}^{2} \mathrm{~K}, \\
& C_{E}=966 \mathrm{~J} /(\mathrm{kgk}), \quad T_{0}=293 \mathrm{~K}, \quad j_{0}=0.196 \times 10^{-4} \mathrm{~m}^{2}, \quad \lambda_{0}=\lambda_{1}=0.37 \times 10^{10} \mathrm{~N} / \mathrm{m}^{2}, \\
& t_{0}=0.01, \quad t_{l}=0.0001, \quad K=252 \mathrm{~J} / \mathrm{msK} .
\end{aligned}
$$

The physical constants for the elastic medium (granite) are given by Bullen (1963) as

$$
\lambda^{e}=0.884 \times 10^{10} \mathrm{~N} / \mathrm{m}^{2}, \quad \mu^{e}=1.2667 \times 10^{10} \mathrm{~N} / \mathrm{m}^{2}, \quad \rho^{e}=2.6 \times 10^{3} \mathrm{Kg} / \mathrm{m}^{3} .
$$

The computations are carried out for the value of non-dimensional time $t=0.2$ in the range $0 \leq y \leq 10$ and on the surface $x=1.0$. The numerical values for normal displacement, normal force stress, temperature distribution and microelongation are shown in Figs 2-5 for mechanical force with magnitude $P_{1}=1.0, h=1, \omega=\omega_{0}+1 \xi, \omega_{0}=0.1, \xi=-0.2$ and $b=1.2$ and $k=2$ for Green-Lindsay (GL) theory. The legend as follows:

(a) thermoelastic microelongated solid(TMS) with $Q=1$ is given by a solid line with dashed symbol $\bullet$.

(b) thermoelastic microelongated solid(TMS) with $Q=10$ is given by a dashed line with centered symbol

(c) thermoelasic solid(TS) with $Q=1$ is given by a dashed line with centered symbol $\boldsymbol{\Delta}$.

(d) thermoelasic solid(TS) with $Q=10$ is given by a dashed line with centered symbol $\times$.

These graphical results represent the solutions obtained by using the generalized theory (G-L theory) by taking $\delta_{1 k}=0, \delta_{2 k}=1$. 
It is observed from Figs 2-5 that the values of normal displacement, normal force stress, temperature distribution and microelongation near the point of application of the source increase with an increase in magnitude of the internal heat source. The values of all the quantities lie in a short range for $Q=1.0$. The values obtained for normal force stress, temperature distribution and microelongation are in the same range. The values of all the quantities converge to zero with horizontal distance.

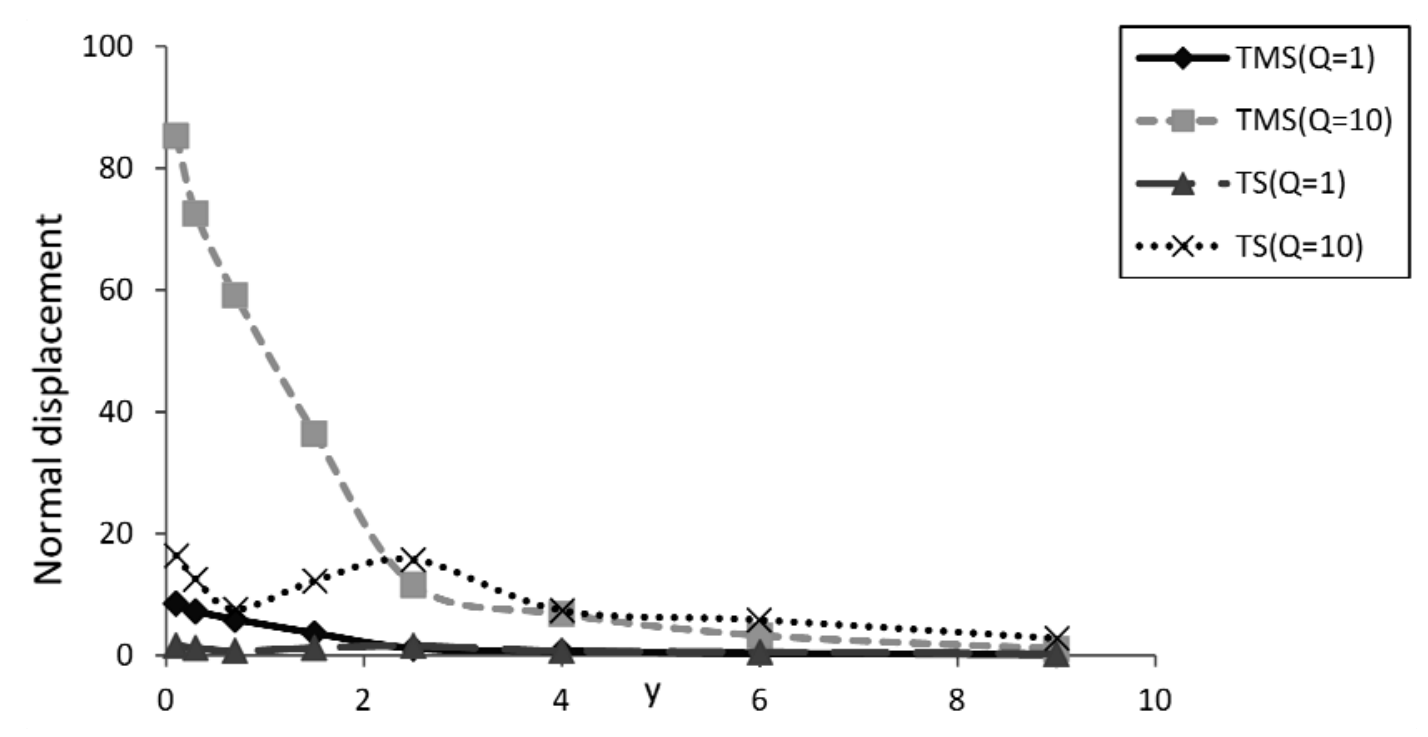

Fig.2. Variation of normal displacement with horizontal distance.

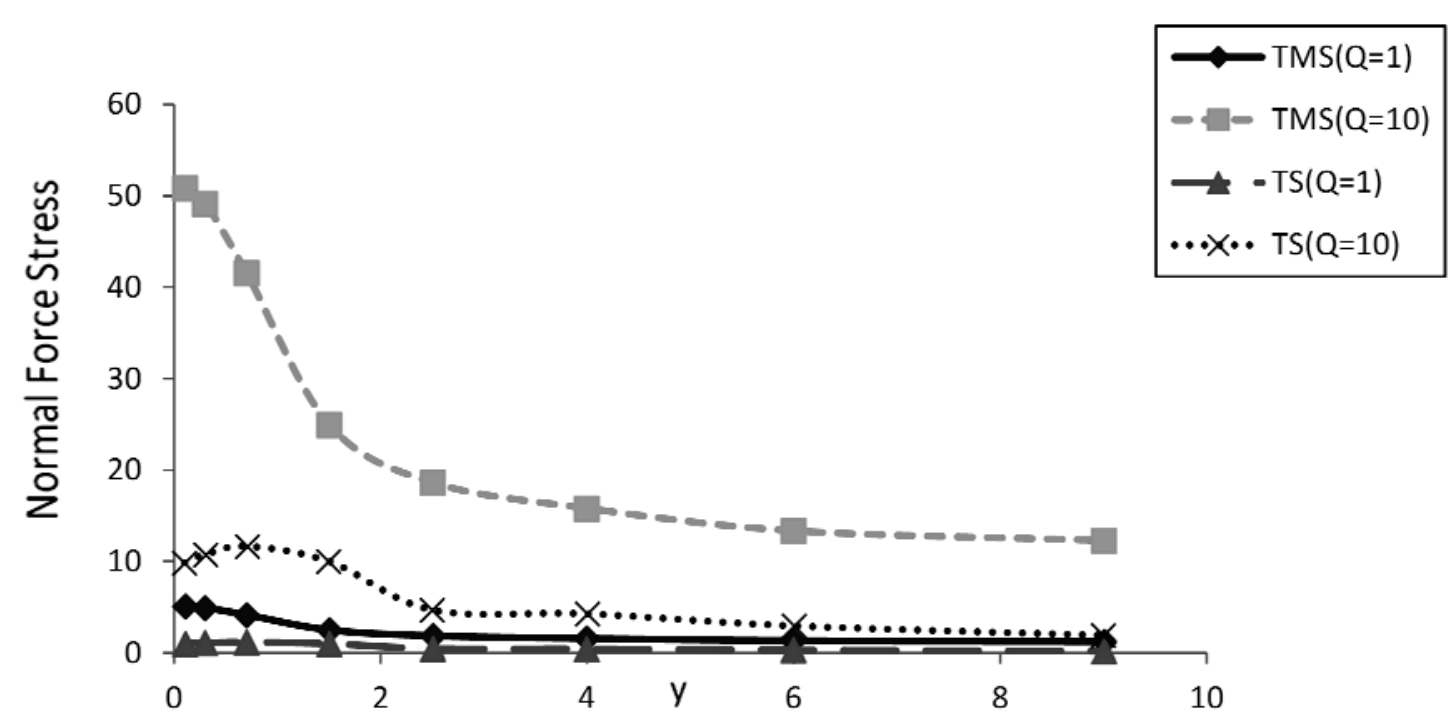

Fig.3. Variation of normal force stress with horizontal distance. 


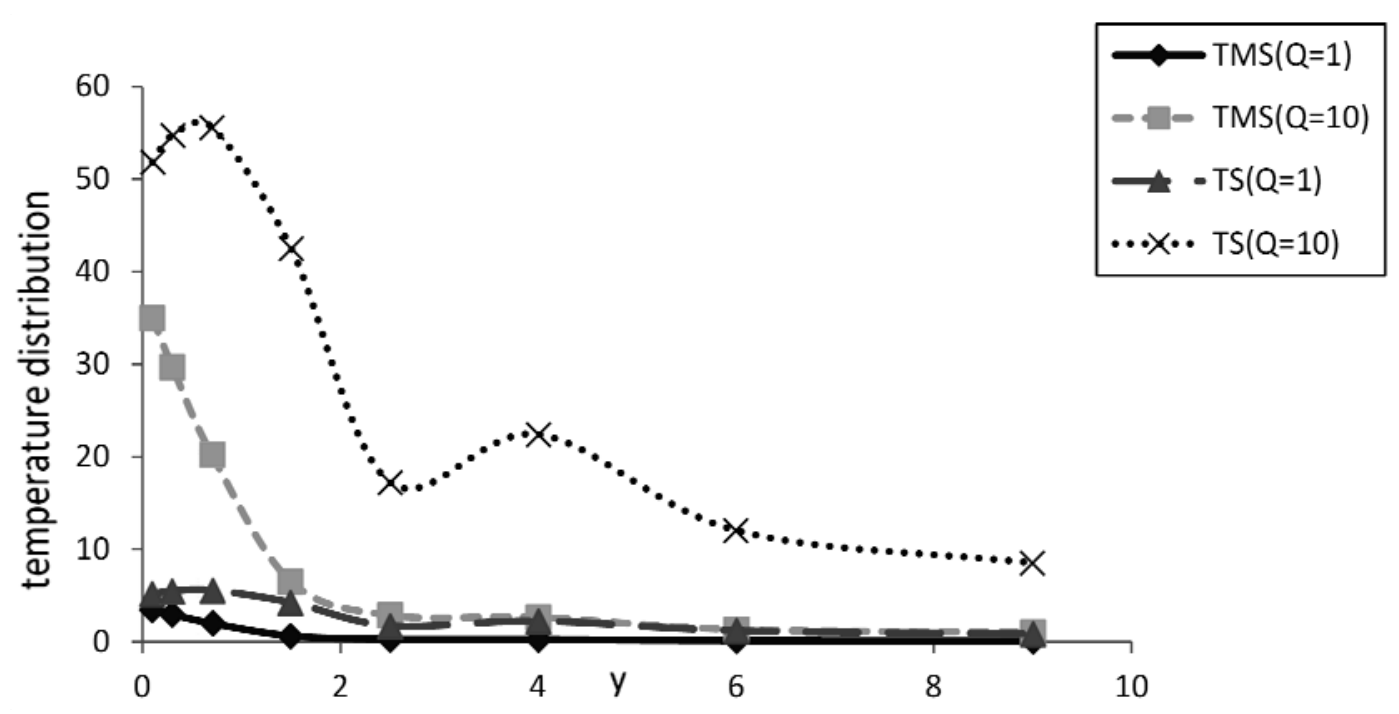

Fig.4. Variation of temperature distribution with horizontal distance.

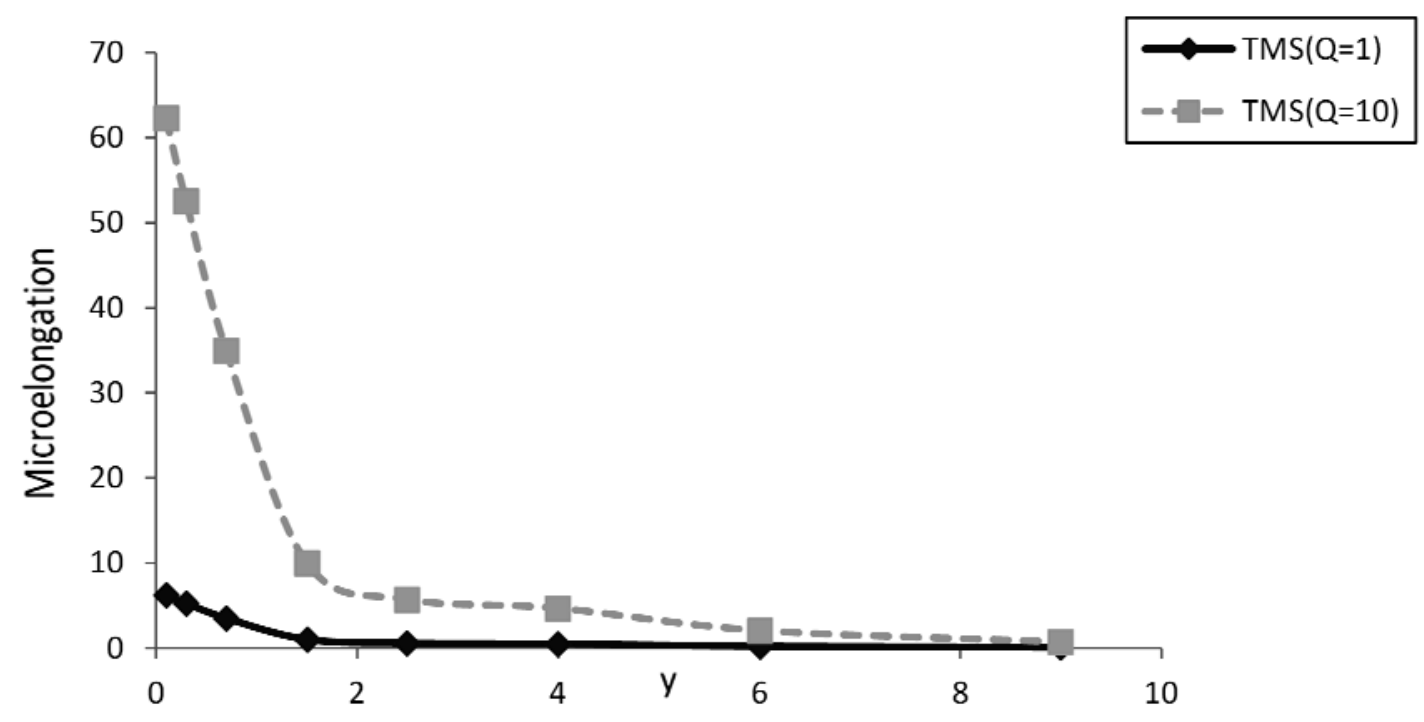

Fig.5. Variation of microelongation with horizontal distance.

\section{Conclusion}

a. Internal heat source and microelongation play a prominent part in the study of deformation of a thermoelastic medium.

b. As expected, the body is deformed to a greater extent with an increase in the magnitude of the internal heat source, near the point of application of the heat source.

c. For a fixed heat source, the value of normal displacement and normal force stress is greater for a microelongated solid, near the application of the source.

\section{Nomenclature}

$a_{0}, \lambda_{0}, \lambda_{1}$ - microelongational constants 
$C_{E}-$ specific heat at constant strain

$K$ - thermal conductivity

$m_{k}$ - component of microstretch vector

$q$ - heat flux

$s=s_{k k} \quad-$ component of stress tensor

$T_{0}$ - reference temperature

$t=\sigma_{k k} \quad-$ microelongational stress tensor

$\boldsymbol{u}$ - displacement vector

$\boldsymbol{u}^{e}$ - displacement vector in elastic medium

$\alpha_{t_{1}}, \alpha_{t_{2}} \quad$ - coefficent of linear thermal expansion

$$
\begin{aligned}
& \beta_{0}=(3+2 \mu) \alpha_{t_{1}} \\
& \beta_{l}=(3+2 \mu) \alpha_{t_{2}}
\end{aligned}
$$

$\lambda^{e}, \mu^{e}$ - Lame's constants in elastic medium

$\rho^{e}-$ density of elastic medium

$\varphi \quad$ - microelongational scalar

\section{References}

Aouadi M. (2006): Thermomechanical interactions in a generalized thermo-microstrech elastic half-space. - Journal of Thermal Stresses, vol.29, pp.511-528.

Barber J.R. (1984): Thermoelastic displacements and stresses due to a heat source moving over the surface of a half plane. - ASME, Transactions, - Journal of Applied Mechanics, vol.51, pp.636-640.

Bullen K.E. (1963): An introduction to theory of seismology. - Cambridge: Cambridge University Press.

Chandrasekharaiah D.S. and Srinath K.S. (1998): Thermoelastic interactions without energy dissipation due to a point heat source. - Journal of Elasticity, vol.50, pp.97-108.

De Cicco S. and Nappa L. (1999): On the theory of thermomicrostretch elastic solids. - Journal of Thermal Stresses, vol.22, pp.565-580.

Deswal S. and Choudhary S. (2008): Two-dimensional interactions due to moving load in generalized thermoelastic solid with diffusion. - Applied Mathematics and Mechanics, vol.29, No.2, pp.207-221.

Dhaliwal R.S. and Singh A. (1987): Micropolar thermoelasticity, Chapter 5, in R.B. Hetnarski(ed.), Thermal Stresses II, Mechanical and Mathematical Methods, ser. 2, North-Holland, Amsterdam.

Dhaliwal R.S., Majumdar S.R. and Wang J. (1997): Thermoelastic waves in an infinite solid caused by a line heat source. - International Journal of Mathematics and Mathematical Sciences, vol.20, No.2, pp.323-334.

El. Maghraby N.M. (2010): A generalized thermoelasticity problem for a halfspace with heat sources and body forces. - International Journal of Thermophysics, vol.31, pp.648-662.

Eringen A.C. (1965): Linear theory of micropolar elasticity. - ONR Techanical report No. 29, School of Aeronautics, Aeronautics and Engineering Science, Purdue University.

Eringen A.C. (1966): A unified theory of thermomechanical materials. - International Journal of Engineering Science, vol.4, pp.179-202.

Eringen A.C. (1970): Foundation of micropolar thermoelasticity. - Courses and Lectures, No.23, CISM, Udine, Springer-Verlag, Vienna and New York.

Eringen A.C. (1971): Micropolar elastic solids with strech. - Ari Kitabevi Matbassi, vol.24, pp.1-18.

Eringen A.C. (1996): Linear theory of micropolar elasticity. - Journal of Mathematics and Mechanics, vol.15, pp.909923. 
Eringen A.C. (1999): Microcontinuum Field Theories. - Vol.1, Foundations and Solids, Springer Verlag, New York.

Eringen A.C. and Suhubi E.S. (1964): Nonlinear theory of simple micro-elastic solids I. - International Journal of Engineering Science, vol.2, pp.189-203.

Eringen A.C. and Kafadar C.B. (1976): In Eringen A.C. (ed.), Continum Physics, Vol. 4, Academic Press, New York.

Ewing W.M., Jardetzky W.S. and Press F. (1957): Elastic waves in layered media. - New York: McGraw Hill.

Green A.E. and Laws N. (1972a): On the entropy production inequality. - Archives of Rational Mechanics and Analysis, vol.45, pp.45-47.

Green A.E. and Lindsay K.A. (1972b): Thermoelasticity. - Journal of Elasticity, vol.2, pp.1-7.

Kiris A. and Inan E. (2007): 3-D vibration analysis of the rectangular microdamaged plates. - In Proc. 8th International Conference on Vibration Problems (ICOVP), India, pp.207-214.

Lord H.W. and Shulman Y. (1967): A generalized dynamical theory of thermo-elasticity. - Journal of the Mechanics and Physics of Solids, vol.15, pp.299-306.

Muller I.M. (1971):The coldness, universal function in thermoelastic bodies. - Rational Mechanics Analysis, vol.41, pp.319-332.

Nowacki W. (1966): Couple stresses in the theory of thermoelasticity III. - Bulletin of the Polish Academy of Sciences Techanical Sciences, vol.8, pp.801-809.

Nowacki W. and Olszak W. (1974): Micropolar thermoelasticity. - In W. Nowacki and Olszak (eds.), Micropolar Thermoelasticity, CISM Courses and Lectures, No.151, Udine, Springer-Verlag, Vienna.

Sarbani C. and Amitava C. (2004): Transient disturbance in a relaxing thermoelastic half-space due to moving internal heat source. - International Journal of Mathematics and Mathematical Sciences, vol.22, pp.595-602.

Sharma J.N. Chauhan R.S. and Kumar R. (2000): Time-harmonic sources in a generalized thermoelastic continuum. Journal of Thermal Stresses, vol.23, No.7, pp.657-674.

Sharma J.N. and Chauhan R.S. (2001): Mechanical and thermal sources in a generalized thermoelastic half-space. Journal of Thermal Stresses, vol.24, No.7, pp.651-675.

Sharma J.N., Sharma P.K. and Gupta S.K. (2004): Steady state response to moving loads in thermoelastic solid media. - Journal of Thermal Stresses, vol.27, No.10, pp.931-951.

Shaw S. and Mukhopadhyay B. (2012): Periodically varying heat source response in a functionally graded microelongated medium. - Applied Mathematics and Computation, vol.128, No.11, pp.6304-6313.

Shaw S. and Mukhopadhyay B. (2013): Moving heat source response in a thermo elastic microelongated solid. Journal of Engineering Physics and Thermophysics, vol.86, No.3, pp.716-722.

Sherief H.H. (1986): Fundamental solution of the generalized thermoelastic problem for short times. - Journal of Thermal Stresses, vol.9, No.2, pp.151-164.

Suhubi E.S. and Eringen A.C. (1964): Nonlinear theory of micro-elastic II. - International Journal of Engineering Science, vol.2, pp.389-404.

Suhubi E.S. (1975): Thermoelastic Solids in Continuum Physics. - New York.

Tauchert T.R. (1971): Thermal stresses in micropolar elastic solids. - Acta Mechanica, vol.11, pp.155-169.

Tauchert T.R., Claus Jr. W.D. and Ariman T. (1968): The linear theory of micropolar thermo-elasticity. - International Journal of Engineering Science, vol.6, pp.36-47.

Youssef H.M. (2010): Generalized thermoelastic infinite medium with spherical cavity subjected to moving heat source. - Computers Mathematical Modelling, vol.21, No.2, pp.211-225. 